\title{
Tie Strength, Optimal Connections, and Distance in Social Networks
}

\author{
David Anthony Buch \\ West Virginia University, dnbuch@mix.wvu.edu
}

Follow this and additional works at: https://researchrepository.wvu.edu/etd

Part of the Quantitative, Qualitative, Comparative, and Historical Methodologies Commons

\section{Recommended Citation}

Buch, David Anthony, "Tie Strength, Optimal Connections, and Distance in Social Networks" (2019). Graduate Theses, Dissertations, and Problem Reports. 7400.

https://researchrepository.wvu.edu/etd/7400

This Thesis is protected by copyright and/or related rights. It has been brought to you by the The Research Repository @ WVU with permission from the rights-holder(s). You are free to use this Thesis in any way that is permitted by the copyright and related rights legislation that applies to your use. For other uses you must obtain permission from the rights-holder(s) directly, unless additional rights are indicated by a Creative Commons license in the record and/ or on the work itself. This Thesis has been accepted for inclusion in WVU Graduate Theses, Dissertations, and Problem Reports collection by an authorized administrator of The Research Repository @ WVU. For more information, please contact researchrepository@mail.wvu.edu. 


\title{
Tie Strength, Optimal Connections, and Distance in Social Networks
}

\author{
David Buch
}

\author{
Thesis submitted \\ to the Eberly College of Arts and Sciences \\ at West Virginia University \\ in partial fulfillment of the requirements for the degree of \\ Master of Science in \\ Applied Mathematics
}

\begin{abstract}
Marjorie Darrah, Ph.D., Chair
Roxann Humbert, Ph.D.

Jessica Deshler, Ph.D.

Department of Mathematics
\end{abstract}

Morgantown, West Virginia

2019

Keywords: Social Networks, Distance, Path Value, Closeness Copyright 2019 David Buch 


\section{ABSTRACT \\ Tie Strength, Optimal Connections, and Distance in Social Networks}

\section{David Buch}

Social network analysis seeks to understand the structure of relationships in networks of actors. As researchers identify structural properties of interest (e.g. vulnerability to network cuts) they introduce measures to quantify the expression of those properties in observed networks. In fact, it is not uncommon that multiple measures are introduced purporting to evaluate a single property. Relative merits of competing measures are not self-evident but the corresponding inferences can conflict, encouraging arbitrary choice among measures and endangering the validity of conclusions. We argue (i) that multiplicity of measures is a necessary consequence of the de rigueur practice of conflating different facets of actor association as "tie strength," and (ii) that distinguishing among distinct facets of tie strength, the primitive unit of Social Network data, implies choices among measures used for other properties. In particular, distinct types of association data (e.g. frequency of contact, emotional depth) must be given distinct mathematical treatment. Hence multiplicity of structural property measures can be reduced to and solved as a problem of multiplicity of treatments for ties. To this end we propose a general framework for evaluation of paths in terms of their ties and introduce novel measures of network connectedness in terms of ties. A key feature of one measure, which we call social conductivity or sconductivity, is its simultaneous accounting for all paths of connection between nodes, which we believe is novel. We discuss the relationship between dimensions of tie strength and appropriate choices from our path-evaluation framework, and show how these choices map bijectively with choices among measures of other structural properties. We conclude by demonstrating the application of the method to the analysis of a network dataset. The methods described have been implemented in an open source software package and published on the Comprehensive R Archive Network. 


\section{Acknowledgements}

I would like to thank my parents

William and Janice Buch

my grandparents

Kenneth and Bennie Buch, and Charles and Joan Ratliff

my committee

Marjorie Darrah, Roxann Humbert, and Jessica Deshler

and the countless other educators in my life. 


\section{Contents}

1 Introduction 1

1.1 Social Network Analysis and Network Property Measures . . . . . . . . . . 1

1.2 Preview of Contributions $\ldots \ldots \ldots \ldots \ldots$

2 Generalized Path Value 3

$\begin{array}{lll}3 & \text { Probabilistic Path Value } & 6\end{array}$

\begin{tabular}{lll}
\hline 4 & Social Conductivity & 7
\end{tabular}

5 gretel: Generalized Path Analysis for Social Networks 8

$\begin{array}{lll}6 & \text { Analysis of the First2 Network } & 10\end{array}$

\begin{tabular}{lll}
\hline 7 & Discussion & 14
\end{tabular}

\begin{tabular}{lll}
\hline 8 & References & 16
\end{tabular}

\begin{tabular}{lr}
\hline A Algorithms & 19
\end{tabular}

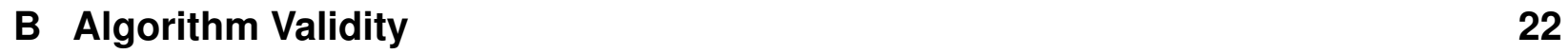

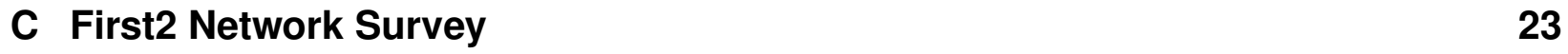




\section{Introduction}

\subsection{Social Network Analysis and Network Property Measures}

In 1934 J.L. Moreno, while studying the group behavioral psychology of children, introduced a new methodology for collecting and representing data, which he called the sociogram [33]. Instead of collecting data to describe individuals, Moreno collected information about the relationships between them, and visualized the individuals and their social links graphically. Moreno later saw this as the birth of sociometry (a precursor to Social Network Analysis [44]) and in 1953 he reflected, "before the advent of sociometry no one knew what the interpersonal structure of a group 'precisely' looked like" [32]. Immediately after its development, the sociogram attracted popular attention [43], and the scheme was usefully extended and refined by researchers throughout the twentieth century [27, 29]. Today, social networks are defined as sets of actors and their relationships, which can be rendered graphically as a network of nodes and edges [44].

Beginning in the 1940s, social network scholars began to recognize the potential for matrices to represent networks numerically, ushering in a flourish of methodological creativity $[4,5,18,26,28,31]$. This was achieved through analogy to matrix representations of graphs in algebraic graph theory, with the row $i$ column $j$ entry describing the connection from actor $i$ to actor $j$. When they represent social network data, these adjacency matrices are called sociomatrices. The matrix form is flexible enough to accommodate networks that are undirected (symmetric) or directed, and binary or valued, depending on what information is collected about the relationships [44].

A typical objective in social network analysis is to evaluate the extent to which a network or its nodes display some abstract structural property. For example, we might hope to understand, as Bott did in 1957 [8], the extent to which individuals in a complex society are social or reclusive. We know $\left(\begin{array}{l}n \\ 2\end{array}\right)$ direct connections are possible in a network, what proportion are present? Putting direct connections aside, what are the worst-casescenario limits of indirect connection? Alternatively, we might be interested in how vulnerable a network is to disruptions. More precisely, we could ask "if a node (or edge) were removed, how would that effect the diameter or average closeness?"

While these questions seem to address well-defined features of the network, there is not always an obvious, unique procedure to obtain quantitative answers. Hence, for each structural property of interest, social network researches have derived and justified quantitative measures. Sometimes, multiple measures are introduced for a single property. We consider some examples:

Countless measures of nodal importance have been introduced under the joint heading of "centrality". Some of the most often cited forms of centrality are betweenness centrality [20,21], closeness centrality [41], graph centrality [24], and stress centrality [42]. Authors have worked to systemize selection among centrality measures. In particular, Borgatti (2005) differentiated among centrality measures by mapping them to implicit 
assumptions about network flow [6]. Borgatti and Everett (2006) take a graph theoretic approach and differentiate centralities according to a four dimensional typology considering 1. type of nodal involvement, 2. type of walk, 3. property of walk studied, and 4. choice of summary measure [7]. Most recently, Agneessens et al. (2017) characterized centralities as measuring either nodal closeness or bokerage, with betweenness centrality a measure of the latter. Moreover, the same authors derived generalized measures of both properties, indexed by a parameter that tunes relative importance of local and remote nodes, that recapitulate previously studied measures of centrality [1].

The units of observation in a network study are direct ties among actors. To probe complex properties, measures operate on "primitive" direct tie data and leverage structural assumptions.

In fact, this ability to combine elementary direct tie data to infer more complex relational states is one of the hallmarks of structural data. For example, we can iteratively multiply a binary sociomatrix by itself, and the first nonzero entry at position $(u, t)$ will be the length of the shortest path from actor $u$ to actor $t$ [44]. It goes without saying that structural property measures must be calculated from algebraic combination of tie data. Frequently path values are found as intermediate steps in the calculation. In all of these cases a decision is made, implicitly or explicitly, as to how we carry out the aggregation of tie values into path values.

Hence, an appealing route to reducing disorder among network property measures is to develop a general framework and typology for path value measures. The selection of an appropriate path value measure at the outset of an analysis removes all ambiguity in selecting among the many path-value-dependent structural property measures later in the analysis.

Why tolerate multiple measures or aggregation strategies at all? Could we not pick just one and move on? We say, "No". There is a long tradition in network analysis of treating tie strength as unidimensional [23]. Tie strength data can represent observations of many different properties of a relationship - frequency of contact, emotional depth, value of shared resources - but it is not unusual, in the analysis stage of a study, to treat the data collected as representing some abstract notion of strength. Granovetter [23] defended the practice on the grounds of "significant correlation among the dimensions of tie strength" so that the distinction is unnecessary and distracting. Others disagree [9]. We take no stance on the debate of whether "tie strength" data can be measured agnostically, but hold a firm belief that different facets regarded as tie strength cannot be aggregated agnostically. This issue will be taken up further in section 2, and has been noted by others [1, 6, 36]. 


\subsection{Preview of Contributions}

The remainder of this article is organized as follows: We begin in Section 2 with a review of $L^{p}$ metrics on vector spaces that provides intuition as we define a family of polynomial generalized path values on networks. We identify traditional path value measures as instances of the polynomial generalized path value. In section 3 , we discuss a probabilistic interpretation of the $p=1$ case, identify problems in the probabilistic model definition, and propose a nonpolynomial metric that does not suffer from the same shortcomings. In section 4, we introduce a new summary measure, which we call social conductivity, that takes into account the total connectedness between nodes along all possible joining paths. In section 5 , we describe our software package that implements these path value and connectedness measures, as well as algorithms that identify paths that are optimal with respect to each of the measures. The optimal path algorithms are particularly useful in finding network diameter and calculating centrality measures. Then, we profile its performance against existing optimal path computational software. Finally, in section 6, we apply our social conductivity measure and other social network analysis techniques to the 2018 First2 Network Survey data, described in the same section. We conclude with in section 7 with summary and discussion.

\section{Generalized Path Value}

The $L^{p}$ norms are used in mathematical analysis as a measure of distance in linear vector spaces [3,40]. The idea is that there is more than one way to sensibly define the length of a vector in terms of its coordinates. Consider a vector $\nu$ whose coordinates sum to 1 . For values of $p$ greater than 1, the length of $\nu$ will be minimized by distributing the coordinate values evenly across dimensions. Specifically, if $\nu$ is $\ell$-dimensional, its norm will be minimized when each coordinate is $\frac{1}{\ell}$. For values of $p$ less than 1 , the opposite is true, the norm of the vector is minimized by concentrating weight in a single coordinate, setting it to 1 and the rest to 0 . In our daily lives, we are accustomed to $p=2$ : that is the heart of the Pythagorean theorem, and the reason why there is brown, trampled grass along the diagonals of campus quadrangles across America. However, while deviations from $p=2$ seem quite exotic, we can think of at least one place in the world where $p=1$.

Usually, if one travels 1 kilometer north and then 1 kilometer east, then he or she will, neglecting the curvature of the earth, wind up roughly $\sqrt{1^{2}+1^{2}} \approx 1.4 \mathrm{~km}$ from where she started. However, in New York City, this fact is not true in a practical sense. Consider that the same walk was taken in Manhattan. The walker may well be $1.4 \mathrm{~km}$ from where they started, but, by the nature of the square blocks, every route home will require they walk $1 \mathrm{~km}+1 \mathrm{~km}=2 \mathrm{~km}$. The combination of distances in two dimensions can be generalized

$$
\operatorname{dis}_{p}\left(\left(x_{1}, y_{1}\right),\left(x_{2}, y_{2}\right)\right)=\left(\left|x_{2}-x_{1}\right|^{p}+\left|y_{2}-y_{1}\right|^{p}\right)^{1 / p}
$$

where $0<p<\infty$. 


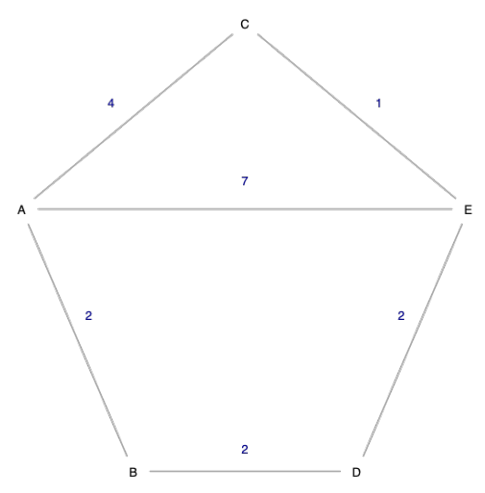

Figure 1: Toy example of a weighted graph

For large $p$, larger components of displacement tend to dominate the net distance, whereas for $p<<1$, the differences in component distance become irrelevant. Accordingly, we can define behavior for $p=\infty$ and $p=0$ by the limiting behavior of Equation 1 .

$$
\begin{gathered}
\operatorname{dis}_{\infty}\left(\left(x_{1}, y_{1}\right),\left(x_{2}, y_{2}\right)\right)=\max \left|x_{2}-x_{1}\right|,\left|y_{2}-y_{1}\right| \\
d i s_{0}\left(\left(x_{1}, y_{1}\right),\left(x_{2}, y_{2}\right)\right)=I\left(\left|x_{2}-x_{1}\right|>0\right)+I\left(\left|y_{2}-y_{1}\right|>0\right)
\end{gathered}
$$

such that $I$ is the indicator function.

We can now generalize $d i s_{p}$ for any number of finite dimensions as

$$
\operatorname{dis}_{p}(a, b)= \begin{cases}\sum\left(I\left(\left|a_{i}-b_{i}\right|>0\right)\right) & \text { for } p=0 \\ \left(\sum\left(\left|a_{i}-b_{i}\right|\right)^{p}\right)^{1 / p} & \text { for } 0<p<\infty \\ \max \left\{\left|a_{i}-b_{i}\right|\right\} & \text { for } p=\infty\end{cases}
$$

where $\left\{a_{i}\right\}$ and $\left\{b_{i}\right\}$ are the coordinates of vectors $a$ and $b$.

Returning to social network analysis, When faced with the task of developing generalized notions of distance in weighted networks, we can follow the example of the $L^{p}$ system for vectors. We borrow the flexible polynomial structure to construct an $L^{p}$-like system for paths in social networks. Now, instead of components of length broken into dimensional coordinates, components of paths are broken into individual ties. To investigate the method and build intuition, consider Figure 1. Suppose the ties represent roads and the nodes represent cities and consider the following scenarios:

$L^{1}$ : The tie values represent toll fees collected along each road. We are interested in finding the optimal path through the network that minimizes total cost of tolls accrued. The value we would like to minimize, and the most useful in comparing paths, is simply 
the sum of the individual components. The optimal path becomes $A B D E$.

$L^{0}$ : Suppose the ties represent physical distances. You would like to transmit a secret message, which will be carried on foot, from town $S$ to town T. At each intervening town in the transmission path, the message will be communicated verbally from one runner to the next. The runners have good memories, but through misinterpretations, each communication introduces some chance of message corruption. The "value" we would like to minimize then is simply the number of runners required, irrespective of the length of their run. We conclude $A E$ is the ideal route.

$L^{\infty}$ : Suppose the ties represent the intensity of security screening along each network. You again need to communicate a message from city $A$ to city $E$, but you no longer have access to runners. Instead, you must carry the message yourself, but you are unwelcome in every other city, and will be arrested if identified. You feel certain you could make it past any number of low-security checkpoints without being caught, but every unit increase in security dramatically increases your risk. Hence, for our purposes, the value that best characterizes the "distance" of each path is the distance of the longest tie. The optimal path becomes $A C B F D E$.

So it appears that these $L^{p}$-like metrics have sufficient flexibility to capture diverse notions of path distance in networks.

From these examples, it should also be noticed that different modeling situations seem to inherently call for different strategies for the accumulation of distances, and these strategies can have a profound effect on how we characterize and compare paths. Note that each of the quantities we considered - toll price, distance, and security measures - could be taken as elements of the "weakness" of a path. The inverses then describe different elements of tie strength.

As described in the introduction, there are many facets of social relationships that are conflated as "tie strength" in the collection of network data [9,23]. Just as in our above examples, where different facets of distance motivated distinct path evaluation schemes, we argue that consideration for the facet of tie strength under consideration should motivate certain choices of path value measures, which in turn guides the selection of other network measures that rely on path values as intermediate steps.

There is an obvious inverse relationship between notions of path distance and path value, and both capture the same information. However, since relational data in networks are usually collected as tie strengths, it seems more desirable that paths would be described in terms of their strength than their weakness, and in units consistent with the original tie strength data. Hence we invert our system of $L^{p}$ distances to obtain

$$
g p v^{w}(i, j)=\min \left(\frac{1}{w_{i h}^{p}}+\cdots+\frac{1}{w_{h j}^{p}}\right)^{-\frac{1}{p}} .
$$


We call this quantity generalized path value. In the $p=0$ case, we obtain inverse binary distance [45]; in the $p=1$ case, we obtain the path value measure proposed by Newman [35]; in the $p=\infty$ case, we obtain Peay's path value [38].

Note the similarity and differences of this result from the generalized distance measure of Opsahl, et al. [36]

$$
d^{w}(i, j)=\min \left(\frac{1}{w_{i h}^{\alpha}}+\cdots+\frac{1}{w_{h j}^{\alpha}}\right) .
$$

Inverting this quantity would obtain something like our generalized path value. However, the units of path value would be entirely inconsistent with the original units of tie strength for any $\alpha$ far from 0 . Further, $\alpha=\infty$, the Peay path value, would not be a viable limiting case.

\section{Probabilistic Path Value}

Within the framework of path value measures introduced in Section 2, the $p=1$ case stands out as particularly intuitive, and is in some sense a safe choice for researchers who are uncertain of how their tie strength data should be accumulated into path values. As it turns out, the $p=1$ measure has another advantage. If the strength of ties emanating at a node are normalized by the sum of the ties, then the new weight of each tie can be interpreted as the probability of a random walker (or resource) egressing from that node to its neighbor along that tie. In fact, the whole network can be reweighted this way (edges will become assymetric, if they weren't already), and so edge weights will come to represent the probability of specific random walk transitions. If this is done, then - as argued by Doyle and Snell [15] - the $p=1$ measure of a path will represent the transition probability of a walker moving sequentially from the beginning of the path to the end.

The availability of a probabilistic interpretation of tie strengths and path values is alluring, but unfortunately the random walk model is not often useful in describing the objects of transmission in social networks. Social movements, for example, are not passed from person to person with a new disciple becoming active only after the first retires. Children limited to transmitting their colds to only a single unlucky victim, and they are not healed the instant their teacher becomes ill. In earnest, the random walk model is better suited as a model for a large game of "hot potato" than many of the interesting payloads of social networks. The issue is that information and disease are transmitted by broadcast, not transfer, so we need a model that accommodates broadcast transmission through networks. In the process we discover that, despite the flexibility of the framework introduced in Section 2, it cannot recapitulate all conceivable path value measures.

To begin with, we need a link between the observed tie strength data and the transmission probability across ties. Since tie strengths are typically reported as positive real 
numbers, not necessarily between 0 and 1 , and because in the broadcast transmission case there is no way to normalize for total transmission probability to equal 1 , we adopt the assumption that tie strengths are recorded in proportion to the odds of broadcast transmission along a node. This assumption should be checked for plausibility before proceeding, but in many cases it appears defensible. For example, if tie strengths record frequency of contact between coworkers, then two coworkers joined by an edge of weight 3 come into contact three times more often than coworkers joined by an edge weight of 1 . It is plausible that this factor of three would carryover into the relative odds that a cold is transferred between either pair of coworkers. If it is decided that the odds scale is appropriate up to proportionality, the researcher can rescale the network weights so that a tie strength of 1 is assigned to all edges along which broadcast transmission is deemed "as likely as not".

Once the issue of identifying tie strengths with transmission odds has been dealt with, the definition of path values that reflect complete path transmission odds proceeds automatically according to the rules of probability. Namely, probabilities can be identified from the transmission odds, and a sequence of independent transmissions has probability equal to the product of transmission probabilities along each step. If we assume that, in general, transmission events are independent, then the optimal path identified with respect to this metric will represent the most likely route of broadcast transmission from a source node to a target node. Fortunately, the optimal path is independent of the precise scale factors used, reducing the reliance of our conclusions on scale assumptions.

Algorithms for the identification of optimal paths with respect to generalized path value and probabilistic path value are detailed in Appendix A.

\section{Social Conductivity}

A frequent object of interest in social network analysis is the extent to which a pair of nodes are connected or disconnected. This question has motivated several measures of path value, which are then used as a scale to identify optimal paths between nodes, and the value of these optimal paths is used as a proxy for the connectedness of a pair [45]. An obvious shortcoming of this strategy is a failure to account for the extent two which two nodes are connected indirectly. If pair $A$ and pair $B$ are close friends, but the members of pair $A$ share numerous other common friends whereas the members of pair $B$ have no mutual friends, it would be inaccurate to describe both pairs as being equally well connected. We would like to simultaneously account for all paths joining pairs of interest. One physical setting in which we have available precise tools for the accounting of multiple paths of transmission is electrical networks. There is an alluring parity between social ties and conductive elements, and there are well developed mathematics for describing total conductivity between points in networks of conductors. Accordingly, we propose social conductivity as a useful measure for summarizing all-paths connectedness between nodes. 
To see a little of how this works, we again consider Figure 1. Using Ohm's law and treating ties as conductive elements with conductivity proportional to tie strength, we can find the social conductivity between nodes $A$ and $E$ as follows:

To obtain the effective resistance of a series of resistors like $A C, A E$, we add their respective resistances. Since we are treating the tie weights as conductivities, we will use harmonic addition instead so we see that path $A C E$ has conductivity $\left(\frac{1}{4}+\frac{1}{1}\right)^{-1}=\frac{4}{5}$. Similarly, path $A B D E$ has conductivity $\left(\frac{1}{2}+\frac{1}{2}+\frac{1}{2}\right)^{-1}=\frac{2}{3}$. Finally, path $A E$ simply has conductivity 7. Next, recall that the effective resistance of parallel resistors is the harmonic sum of the component resistances. Therefore, working with conductivities, we simply take the sum of the component conductivities. Therefore, the conductivity between nodes $A$ and $E$ is the sum of the conductivity of the three paths joining these nodes, $\frac{4}{5}+\frac{2}{3}+7=\frac{127}{15} \approx 8.5$.

The key properties of social conductivity are that no path is more conductive than its least conductive tie, and the total conductivity of two disjoint parallel paths is the sum of their individual conductivities.

To account for all paths in highly complex networks, matrix techniques are required. The calculations for these networks is described in the manual for the $R$ package for electrical engineering ResistorArray [25]. Notice that, in its current definition, social conductivity can only operate on symmetric networks.

\section{5 gretel: Generalized Path Analysis for Social Networks}

The software package gretel is an open source extension to the statistical programming language $\mathbf{R}$ [39]. It was developed as a companion to this thesis, and provides user-friendly functions for analyzing paths in both the generalized path value and probabilistic path value settings. Further, gretel features functions that summarize all-pairs connectedness as social conductivity or optimal path values with respect to generalized path value or probabilistic path value.

Given a sociomatrix and a valid user specified path, the gpv and ppv functions calculate the generalized path value and the probabilistic path value, respectively, according to their definitions in sections 2 and 3 . The functions opt_gpv and opt_ppv identify optimal paths from a user-specified source to a user-specified target under either framework of path value, and all_opt_gpv and all_opt_ppv identify optimal paths among all pairs.

The proximity function summarizes all-pairs connectedness. It takes a sociomatrix as its input and returns a matrix encoding social conductivity or optimal path value for each directional pair as an "effective proximity" among nodes. This transformed matrix could be used for clustering or other unsupervised learning methods that rely on a notion 
of distance in graphs.

Finally, functions binary_distance, flament_path_length, peay_average_path_value, and flament_average_path_length calculate historically useful measures of path value and distances, as described in Flament (1963), Peay (1980), and Yang \& Knoke (2001) [17, 38, 45].

A feature of the software package that is not introduced in our earlier theory discussions is the ability to account for individual traits of nodes. The generalized path value family of functions take an optional node_costs argument that indicates any penalty associated with the use of one or more of these nodes. Traversal along a path through a node with a nonzero cost will accrue that cost of that node as if it were an edge length. However, a nonzero terminal node cost will not be leveraged against the path when calculating path value. It is easy to see that the decision to include or exclude terminal node costs has no consequence on the shortest path problem.

Similarly, the probabilistic path value family of functions take the optional argument odds_scale (equal for all nodes) or even odds_scale_by_node. Without these parameters, the probabilistic path value functions make the assumption that a tie strength of 1 reflects even odds of transmission for all nodes in the network. Of course, true transmission odds will depend on actor appraised urgency of transmission, but even neglecting that variability, if transmission odds were relatively consistent across a range of urgencies (or virulence), it is possible that two people could express the same intensity of friendship but, depending on degree of extroversion, exhibit differential transmission odds to their peers. Therefore, tie strengths are divided by their source node's odds scale before interpretation or combination in the probabilistic perspective. Most likely, these parameters will only be identifiable in cases where actual network communication data is available to estimate latent odds scales. Importantly, any odds scale uniform across all nodes will have no influence on the shortest path problem. Thus, if we believe the scale is roughly constant and are only concerned with identifying optimal paths, the parameter can be ignored.

Shortest path problems, and especially all-pairs shortest path problems are well known for their computational expense [22]. In all our algorithms, detailed in the appendices, we rely essentially on the seminal shortest path algorithm of Dijkstra [14]. To maximize performance and allow for the analysis of large $\left(n>10^{3}\right)$ networks, we implemented these algorithms in the compiled language $\mathrm{C}_{++}$, ported to $\mathrm{R}$ through the Rcpp package [16]. The social conductivity calculations rely on the principles of resistor array analysis, a modern topic in electrical engineering. For these calculations gretel invokes the ResistorArray package [25].

In Figure 2 we exhibit the computational performance of our package compared to the popular social network analysis package tnet [37]. From these data it is clear that our software is competitive with tnet in solving shortest path problems on small networks but is linearly more expensive. This extra cost is thought to be due to our choice of data structure, as Fibonacci Heaps are known to be more efficient for this problem than plain 

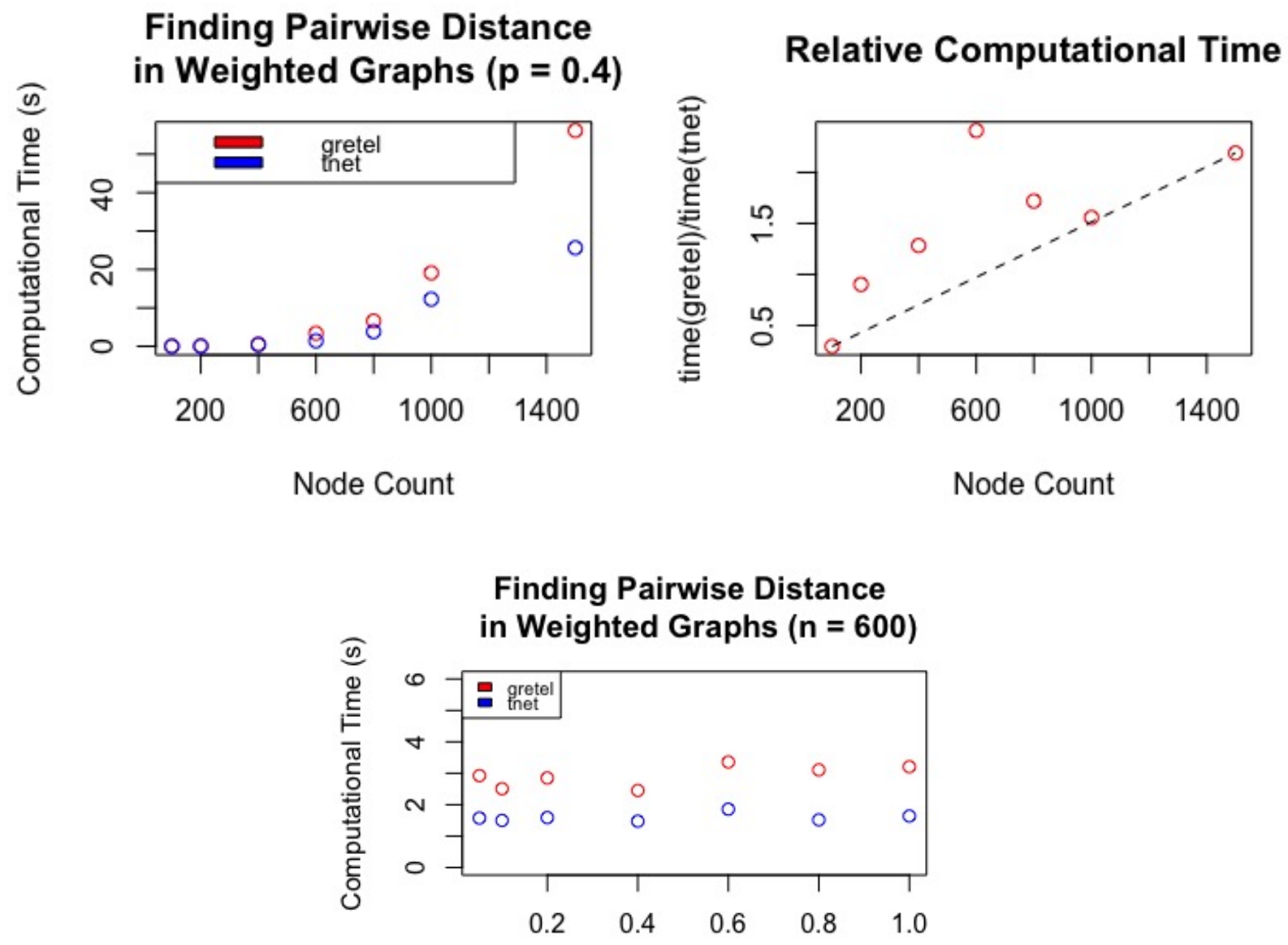

Edge Probability

Figure 2: Performance data for the gretel software package

arrays [19].

To learn more about gretel and how it can be used, see the package website [11] and in particular the package vignette [12].

\section{Analysis of the First2 Network}

The First2 Network is a consortium of students, educators, and other stakeholders founded in 2016 with the objective of improving the retention rate of undergraduate STEM (Science, Technology, Engineering, and Mathematics) majors, particularly first generation students from rural areas of West Virginia [34]. Members of First2 are optionally associated with one or more subgroups called working groups. In 2018, the First2 Network was awarded grant funding from the National Science Foundation in part to expand the reach 
and capacity of the First2 program [34]. To carry out its mission of improving persistence in STEM fields, members of the network need to understand the role of student-faculty and student-student relationships in student success. Further, to make progress expanding its scope and accomplishing the goals articulated in its grant funding, the network must extend its reach without disintegrating. Collecting structural data on the First2 Network itself provides useful information in addressing both of these tasks. In 2019, a survey was conducted among all active members of the First2 Network. Of just over 60 people contacted to complete the survey, 25 responses were obtained generating 48 unique names. The First 2 Network Survey, including the name generator, is reproduced in full in Appendix C. Currently there are plans to administer the survey annually so that network time series can be obtained. The anonymized 2019 network is visualized in Figure 3.

Once obtaining year over year network data, we hope to answer questions about the growth of the network, causal effects of interventions on the network [10], and the relationship between student outcomes and their network status. For now, we are able to answer slightly less ambitious, static-time questions: How well connected are typical dyads in the network? How vulnerable is the network? Does vulnerability/strength vary among working groups?

In Table 1 we summarize some preliminary results towards these questions, with the caveat that there was non-negligible non-response (48 out of an estimated $60-70$ members were identified) and it is not clear whether unavailable data were missing at random.

\begin{tabular}{|l|l|l|l|}
\hline Network & Isolated Nodes & Conductivity & Robustness \\
\hline First2 Net & 0 & 2.8 & 0.86 \\
Cap Build & 0.5 & 0.37 & 0.09 \\
Sum Imm & 0.2 & 1.4 & 0.9 \\
Col Ready & 0.12 & 1.0 & 0.75 \\
Fac/Stud & 0.18 & 0.87 & 0.63 \\
\hline
\end{tabular}

Table 1: Summary of the 2019 First2 Network Alliance

The data, meant to be considered together and calculated year over year, include three measures of connectedness evaluated on the complete network and each of the working groups. The isolated nodes column reports a percent of isolated nodes for each network. For potentially disconnected networks, this is more informative that network diameter, since the latter is set to infinity for all disconnected networks by default. Since every actor in the network either completed the name generator survey or was identified by another actor, there are no isolated nodes in the complete network. However, the capacity building working group stands out as being particularly fragmented. The four working group networks as visualized in Figure 4

The conductivity column in Table 1 reports the mean pairwise social conductivity for the nodes in each network. Robustness reports the average difference between the pair- 


\section{First2 STEM Alliance}

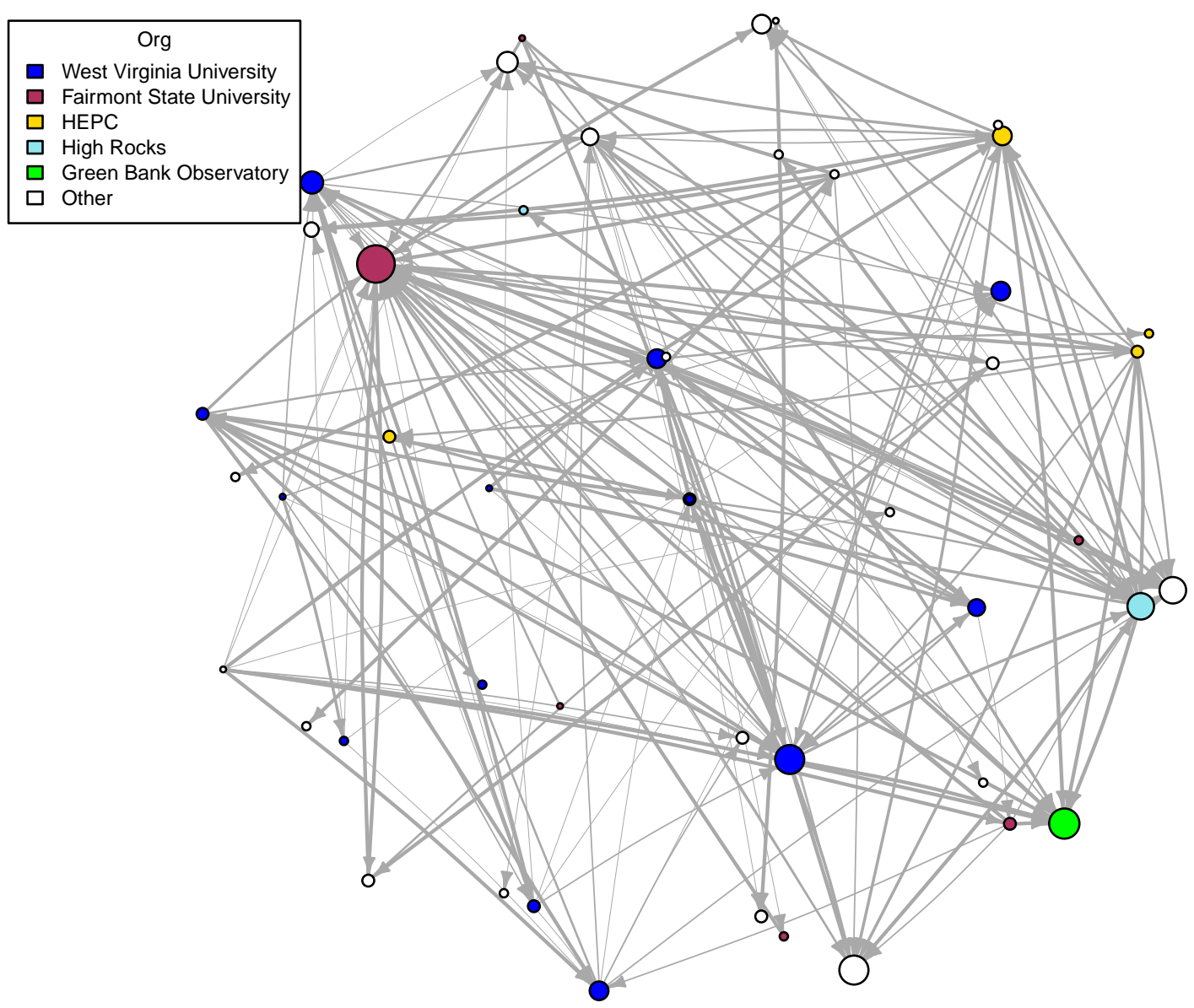

Figure 3: The First2 Network Alliance in 2019 
First2 Summer Immersion Working Group

First2 Capacity Building Working Group

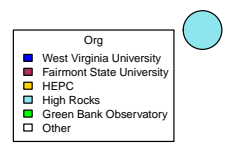

$\bigcirc$

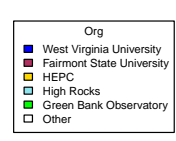

$\bigcirc$

블
HigC Rocks
Gigeen Bank Observatory
G Other

0
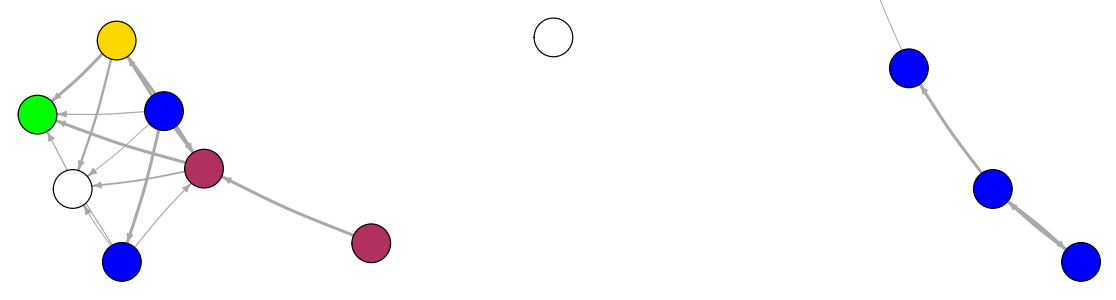

First2 Faculty-Student Engagement Working Group

First2 College Readiness Working Group

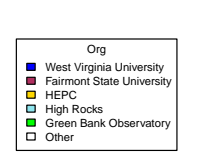

$\bigcirc$

\begin{tabular}{l} 
Fairmont State University \\
HEFC \\
H High hocks \\
Green Bank Observatory \\
G Other \\
\hline
\end{tabular}

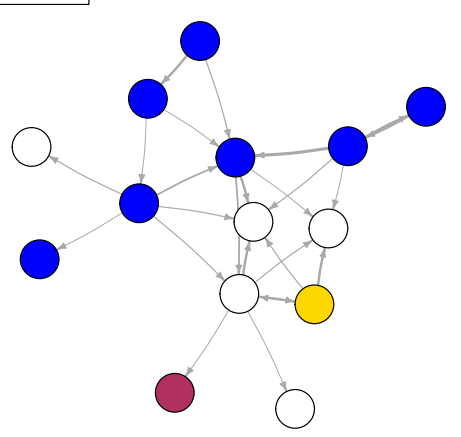

$\bigcirc$
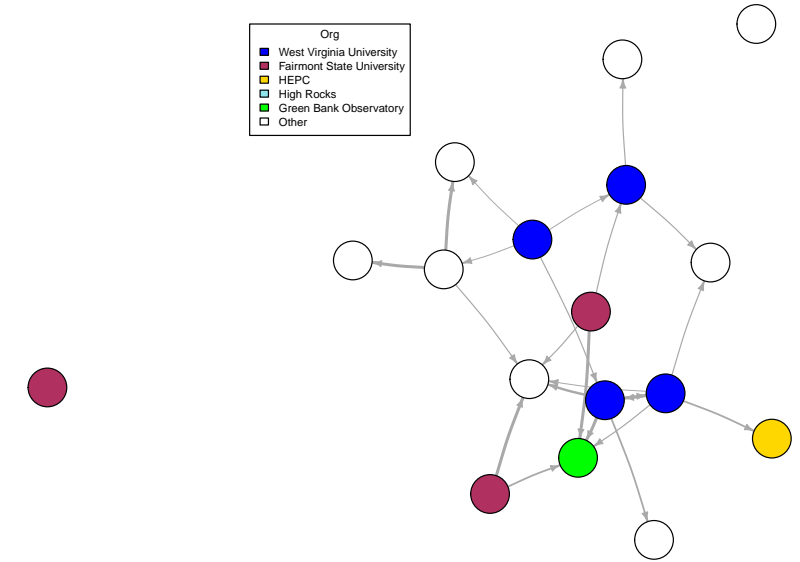

0

$\bigcirc$

Figure 4: Working groups within the First2 Network Alliance in 2019 
First 2 Network 2019

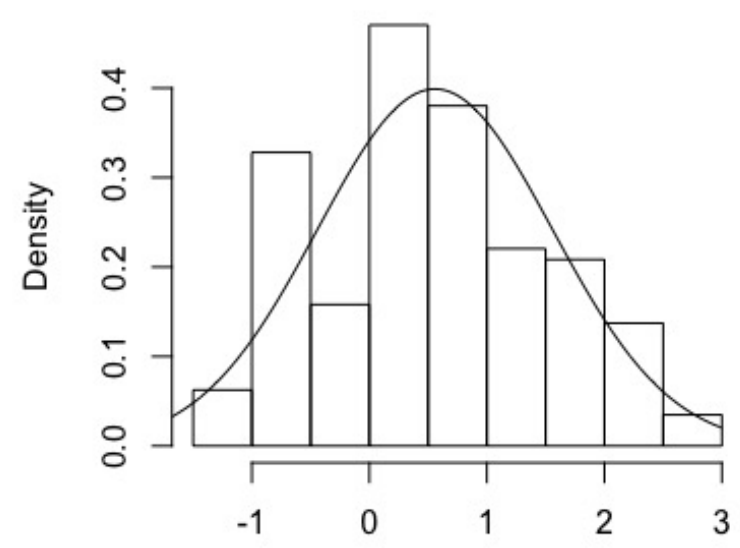

Log Dyadic Intensity
First 2 Network 2019

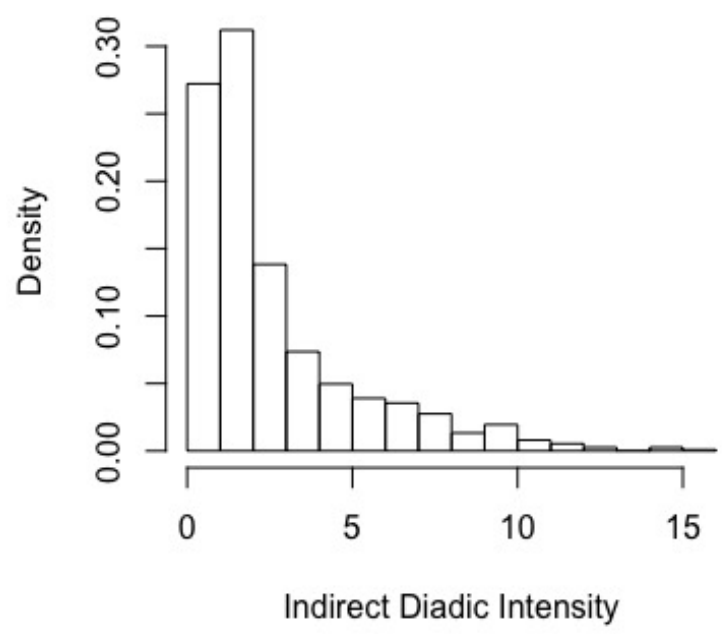

Figure 5: Logscale histograms of social conductivity and robustness of dyads.

wise conductivity and pairwise direct tie strengths. The robustness measure is meant to capture the extent to which strong indirect connections are available between actors, in case direct ties are lost or unavailable. On average, despite having a higher proportion of isolated nodes, the Summer Immersion working group exhibited greater conductivity and robustness than the College Readiness working group. This indicates that, when they exist, relationships within the Summer Immersion working group are stronger and more effectively intertwined than in the College Readiness working group. This conclusions can be assessed visually in Figure 4.

Finally, in coming years we would like to make simple, statistically principled statements about network development. It is known that network traffic are well fit by the lognormal distribution (see, for example, [2]). Therefore, we believe that the lognormal distribution may be a satisfying model for the social conductivity of dyads. See Figure 5 as evidence. Using the normal model for the log of social conductivity, we can invoke a standard two-sample t-test to assess the significance of growth or decline in the mean social conductivity, calculated for each working group in 1 .

\section{Discussion}

We have made an argument for the importance of path value in the calculation of graph properties in social network analysis, in particular, arguing that some disagreements in measures of network properties stem from disagreements in the evaluation of paths. 
If a strategy for path valuation is chosen carefully early in an analysis, it will imply rational selections among measures for more complex properties. This is desirable because a choice of path value measure can usually be made more easily compared to the measures of more complex properties, because the relative implications of different path value measures are more obvious. The decision can be informed by considering which facet of tie strength is encoded by the edge weights. Then, intuition can argue for the correct mathematical treatment of that facet. Another advantage of selecting among property measures based on a choice of path value measure is that consolidating subjective selections to a single explicit choice highlights analytical assumptions and facilitates peer review.

We have introduced a general polynomial scheme for path value calculation, a nonpolynomial path value measure meant to model transmission odds, and a summarization measure, social conductivity, which captures all-paths connectedness among two actors in a network. We derived and implemented efficient algorithms for identifying optimal paths under any of the paradigms. We identified existing measures as specific instances of the polynomial scheme, argued for the necessity of multiple measures as a consequence of multiplicity of facets of tie strength, and recommended appropriate path value measures to be applied to certain facets of tie strength.

Finally, we introduce a publicly available software package that quickly calculates the measures described and can rapidly identify optimal paths with respect to any of the path value measures described.

Directions for future work include large scale case studies and extension of the probabilistic path value model to account for differential message urgency and rate of transmission. 
Tie Strength, Optimal Connections, and Distance in Social Networks

\section{References}

[1] E. Agneessens, Borgatti, Geodesic based centrality: unifying the local and the global, Social Networks, (2017).

[2] I. Antoniou, V. IVAnov, V. V. IVAnov, ANd P. Zrelov, On the log-normal distribution of network traffic, Physica D: Nonlinear Phenomena, 167 (2002), pp. 72 85.

[3] S. AXLER, Linear Algebra Done Right, Undergraduate Texts in Mathematics, Springer, 2015.

[4] R. Bock AND S. HUSAIN, An adaptation of holzinger's b-coefficients for the analysis of sociometric data, Sociometry, (1950).

[5] __ Factors of the tele: A preliminary report, Sociometry, (1952).

[6] S. P. Borgatti, Centrality and network flow, Social Networks, 27 (2005), pp. 55 71.

[7] S. P. Borgatti AND M. G. Everett, A graph-theoretic perspective on centrality, Social Networks, 28 (2006), pp. $466-484$.

[8] E. BotT, Family and Social Network, Tavistock: London, 1957.

[9] M. E. BRAshears AND E. QuinTANE, The weakness of tie strength, Social Networks, 55 (2018), pp. 104-115.

[10] K. H. Brodersen, F. Gallusser, J. Koehler, N. Remy, and S. L. Scott, Inferring causal impact using bayesian structural time-series models, Ann. Appl. Stat., 9 (2015), pp. 247-274.

[11] D. BuCH, gretel website, (2019).

[12] D. A. Buch, gretel: Generalized Path Analysis for Social Networks, 2019.

[13] G. CSARDI AND T. Nepusz, The igraph software package for complex network research, InterJournal, Complex Systems (2006), p. 1695.

[14] E. W. Dijkstra, A note on two problems in connexion with graphs, Numerische Mathematik, 1 (1959), p. 269.

[15] P. Doyle AND J. L. SNeLL, Random walks and electric networks, the Mathematical Association of America, 2000.

[16] D. Eddelbuettel And R. Françols, Rcpp: Seamless $R$ and $C++$ integration, Journal of Statistical Software, 40 (2011), pp. 1-18.

[17] C. Flament, Applications of Graph Theory to Group Structure, Prentice-Hall, Englewood Cliffs, 1963. 
[18] E. ForSYTH AND L. KATZ, A matrix approach to the analysis of sociometric data: Preliminary report, Sociometry, (1946).

[19] M. L. Fredman ANd R. E. TARJAN, Fibonacci heaps and their uses in improved network optimization algorithms, J. ACM, 34 (1987), pp. 596-615.

[20] L. Freeman, A set of measures of centrality based on betweenness, Sociometry, (1977).

[21] _ Centrality in social networks: conceptual clarification, Social Networks, (1979).

[22] Z. GotTHILF AND M. LeWENSteIn, Improved algorithms for the $k$ simple shortest paths and the replacement paths problems, Information Processing Letters, 109 (2009), pp. 352-355.

[23] M. S. Granovetter, The strength of weak ties, American Journal of Sociology, 78 (1973), pp. 1360-1380.

[24] HAGE AND HARARY, Eccentricity and centrality in networks, Social Networks, (1995).

[25] R. K. S. HANKIN, Resistor networks in r: introducing the 'resistorarray' package, $\mathrm{R}$ News, 6 (2006).

[26] F. HARARY, On the notion of balance of a signed graph, Michigan Mathematical Journal, (1953).

[27] G. S. G. B. R. H. M. C. J. S. M. M. J. G. J. WoelfEL, E.L. FinK, Galileo: A program for metric multidimensional scaling, (1977).

[28] L. KATZ, On the matric analysis of sociometric data, Sociometry, (1947).

[29] A. KLOVDAHL, View-net: A new tool for network analysis, Social Networks, (1986).

[30] D. KNOKE AND S. YANG, Social Network Analysis, vol. 154 of quantitative applications in the social sciences, Sage Publications, Inc, 2 ed., 2007.

[31] R. Luce AND A. PerRY, A method of matrix analysis of group structure, Psychometrika, (1949).

[32] MORENO, Who shall survive? foundations of sociometry, group psychotherapy and psychodrama, (1953).

[33] J. L. Moreno, Who shall survive?: A new approach to the problem of human interrelations, vol. 58 of Nervous and mental disease monograph series, Nervous and Mental Disease Publishing Co., 1934.

[34] F. Network, The First2 Network About Page, 2019.

[35] M. NewmAN, Scientific collaboration networks, ii, shortest paths, weighted networks, and centrality, Physical Review: E, (2001). 
[36] S. OpsahL, AGneEssens, Node centrality in weighted networks: Generalizing degree and shortest paths, Social Networks, (2010).

[37] T. OPSAHL, Structure and Evolution of Weighted Networks, University of London (Queen Mary College), London, UK, 2009.

[38] E. R. PEAY, Connectedness in a general model for valued networks, Social Networks, 2 (1980), pp. 385-410.

[39] R Core Team, R: A Language and Environment for Statistical Computing, R Foundation for Statistical Computing, Vienna, Austria, 2019.

[40] W. Rudin, Principles of Mathematical Analysis, McGraw Hill Education, 2018.

[41] SABIDUSSI, The centrality index of a graph, Psychometrika, (1966).

[42] SHIMBEL, Structural parameters of communication networks, Bulletin of Mathematical Biophysics, (1953).

[43] T. N. Y. TIMES, Emotions mapped by new geography, (1933).

[44] S. WASSERMAN AND K. FAUST, Social network analysis: Methods and applications, vol. 8 of Structural Analysis in the Social Sciences, Cambridge University Press, New York, 1994.

[45] S. YANG AND D. KNOKE, Optimal connections: strength and distance in valued graphs, Social Networks, 23 (2001), pp. 285-295. 


\section{A Algorithms}

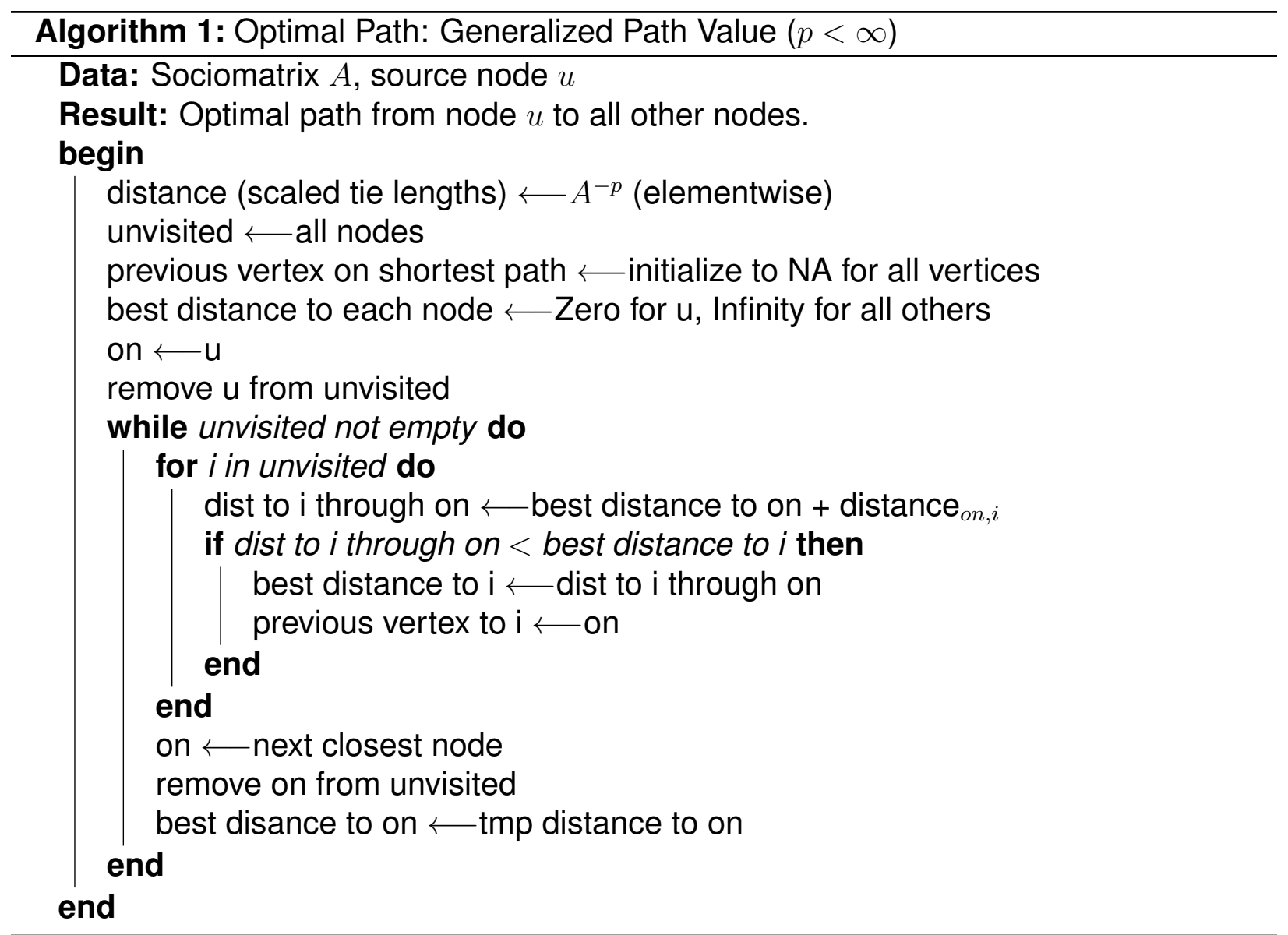

Note that we raise tie "lengths" to the power $p$ but never evaluate the $p^{\text {th }}$ root of the final path lengths. This is because Dijkstra's algorithm only requires we know relative lengths of paths. Rooting the sum of the calculated distances is monotonic, and would not change our conclusions. We just need to be sure to take the root before reporting the optimal path's generalized value. 


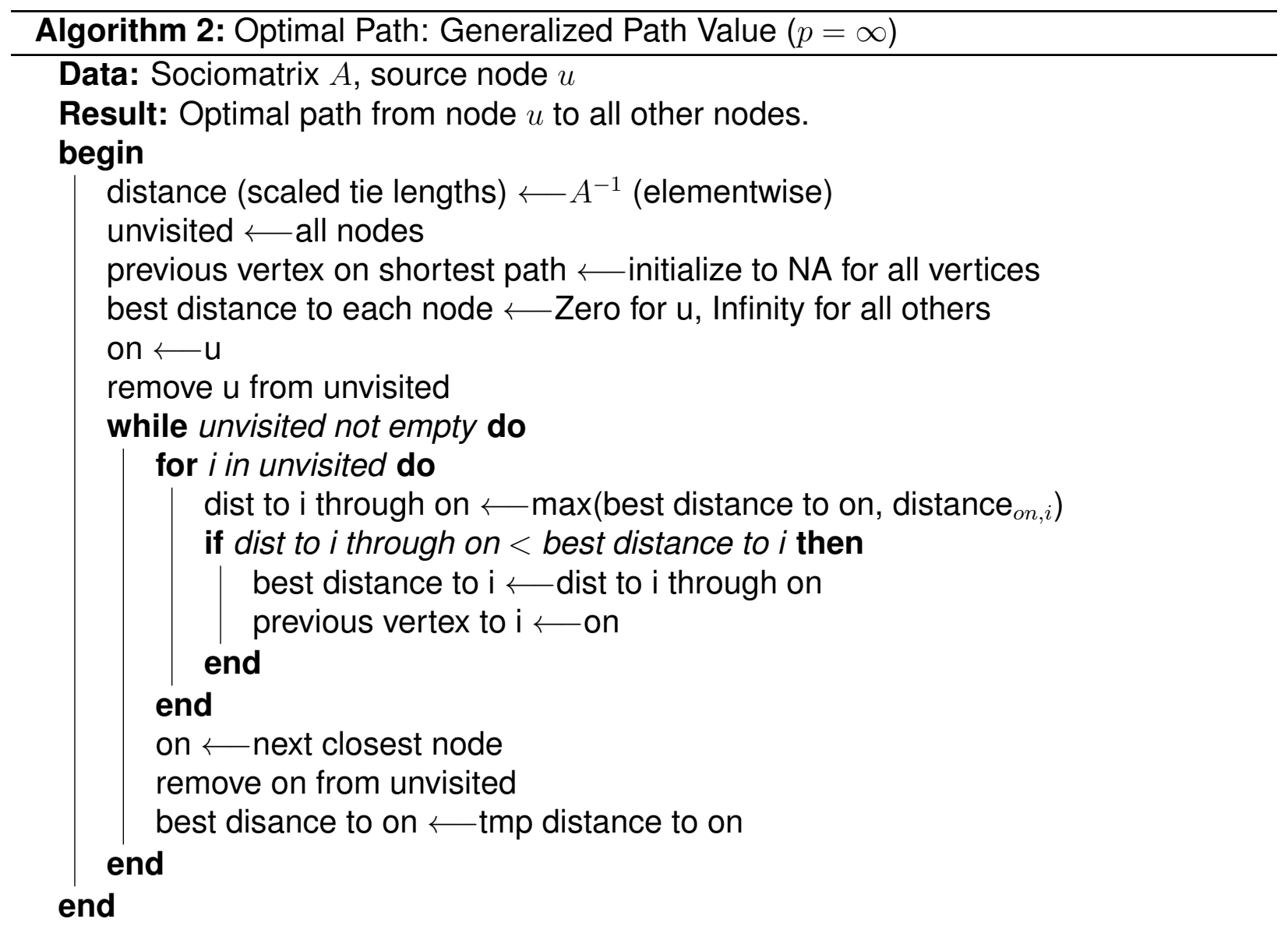

Note here that the tie "lengths" are raised to the first power. The choice is arbitrary, though, and any monotonic function will do, since $\max (f(a), f(b))=\max (a, b)$ for any monotonic $f$. 


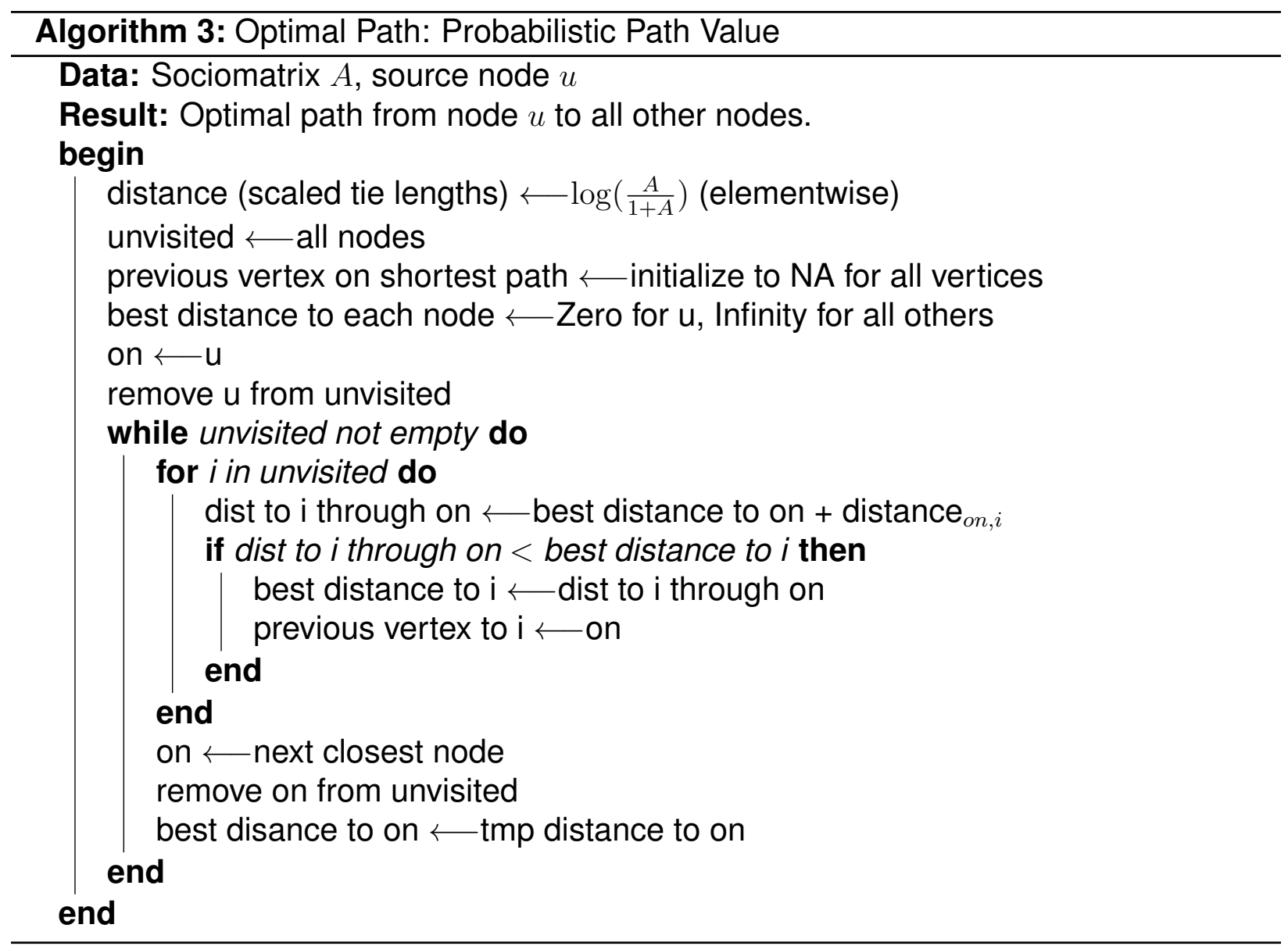

See Appendix B for proof of validity. 


\section{B Algorithm Validity}

Consider the family $\mathcal{P}_{A B}$ of all possible paths between vertices $A$ and $B$. We are interested in an efficient algorithm for solving

$$
\max _{P \in \mathcal{P}_{A B}} \text { Transmission } \operatorname{Odds}(P)
$$

Or, since

$$
f(x)=\frac{x}{1+x}
$$

is monotonic, we can equivalently solve

$$
\max _{P \in \mathcal{P}_{A B}} \operatorname{Pr}(P),
$$

where $\operatorname{Pr}(P)$ is the transmission probability of path $\mathrm{P}$. Here we propose a solution that is based at its heart on Dijkstra's shortest path algorithm. Importantly, though, these algorithms rely on the additive concatenation of distances (Length of path $P a b c=P a b+P b c$ ). Probabilities however, are multiplicatively concatenated. Recall, however, that $\log (a * b *$ $\cdots * k)=\log (a)+\log (b)+\cdots+\log (k)$ Therefore,

$$
\log (\operatorname{Pr}(P))=\log \left(p_{12}\right)+\log \left(p_{23}\right)+\log \left(p_{34}\right)
$$

. Thus, log probabilities have the desirable property of additive concatenation. Further, log is a monotonic transform, so $\log \left(\operatorname{Pr}\left(P_{a}\right)\right)>\log \left(\operatorname{Pr}\left(P_{b}\right)\right)$ implies $\operatorname{Pr}\left(P_{a}\right)>\operatorname{Pr}\left(P_{b}\right)$. Therefore, it is appropriate that we instead consider the optimization

$$
\max _{P \in \mathcal{P}_{A B}} \log (\operatorname{Pr}(P))
$$

With a final modification, this becomes

$$
\min _{P \in \mathcal{P}_{A B}}-\log (\operatorname{Pr}(P))
$$

Which is solvable in $\mathcal{O}\left(V^{2}\right)$ time by Dijkstra's shortest path algorithm [14]. 
Tie Strength, Optimal Connections, and Distance in Social Networks

\section{First2 Network Survey}




First2 Network Survey $\vee$
Survey

First2 Network Survey

\begin{tabular}{|c|c|c|}
\hline Q1 & Principal Investigator: & Dr. Gay Stewart \\
\hline & Department: & WVU Department of Mathematics \\
\hline & Protocol Number: & 1807185583 \\
\hline & Study Title: & First2 Network Survey \\
\hline$i Q$ & $\begin{array}{l}\text { Co-Investigator(s): } \\
\text { Sponsor (if any): }\end{array}$ & $\begin{array}{l}\text { Dr. Roxann Humbert, Dr. Marjorie Darrah } \\
\text { NSF INCLUDES Grant HRD-1834569 }\end{array}$ \\
\hline
\end{tabular}

Contact Persons

In the event you experience any side effects or injury related to this research, you should contact Dr. Marjorie Darrah at (304) 293-2011 or Marjorie.Darrah@mail.wvu.edu. You should also contact Dr. Darrah if you have any questions, concerns, or complaints about this research.

For information regarding your rights as a research subject, to discuss problems, concerns, or suggestions related to the research, to obtain information or offer input about the research, contact the Office of Research Integrity and Compliance at (304) 293-7073.

In addition if you would like to discuss problems, concerns, have suggestions related to research, or would like to offer input about the research, contact the Office of Research Integrity and Compliance at 304-293-7073.

Introduction

You have been asked to participate in this research study, which is described in your email invitation. This study is being conducted by Dr. Marjorie Darrah and Dr. Roxann Humbert in the Department of Mathematics at West Virginia University with funding provided by NSF INCLUDES grant HRD-1834569.

Purpose(s) of the Study

The purpose of the study is to characterize and understand how evolving connections among faculty, students, and allies of higher education can be leveraged to improve outcomes for first generation college students from rural WV. Further details are provided in the preamble to Question 9.

Description of Procedures

This study involves the completion of the attached questionnaire and will take approximately 10-15 minutes for you to complete. You do not have to answer all the questions. You have the opportunity to see the questionnaire now before signing this consent form.

Discomforts

There are no known or expected risks from participating in this study, except for the mild frustration associated with answering the questions.

Benefits

You may not receive any direct benefit from this study. The knowledge gained from this study may eventually benefit others.

Financial Considerations

There are no special fees or inducements for participating in this study. Confidentiality

Any information about you that is obtained as a result of your participation in this research will be kept as confidential as legally possible. Your research records and test results, just like hospital records, may be subpoenaed by court order or may be inspected by the study sponsor or federal regulatory authorities (including the FDA if applicable) without your additional consent.

In addition, there are certain instances where the researcher is legally required to give information to the appropriate authorities. These would include mandatory reporting of infectious diseases, mandatory reporting of information about behavior that is imminently dangerous to your child or to others, such as suicide, child abuse, etc. 


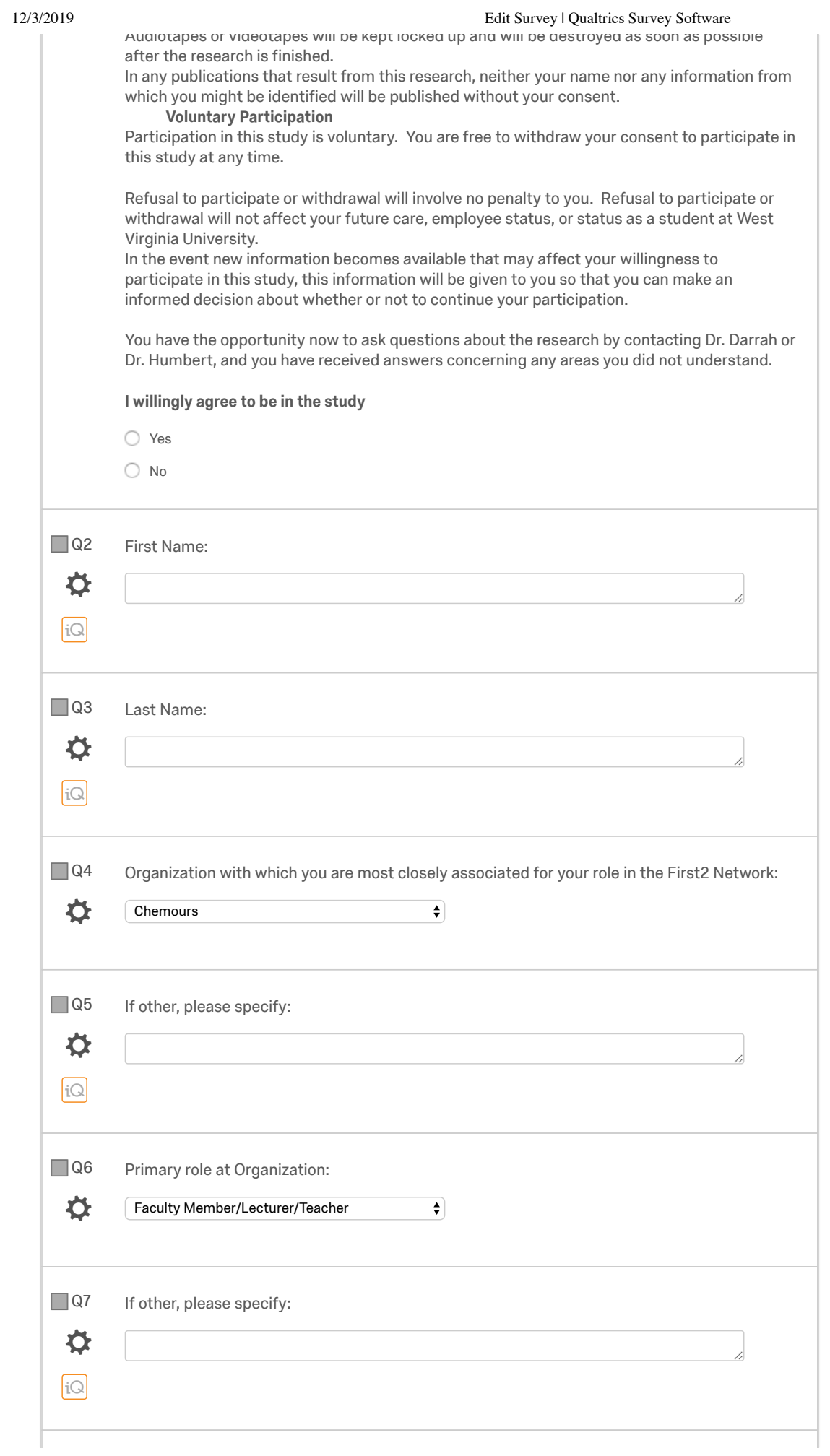


Social network studies seek to observe, characterize, and draw conclusions from patterns of connections among associates in some setting of interest. Examples range from patterns of D. play among school children (Guralnick, et al., 2009) to strategic alliance and competition among corporations on the international economic stage (Osborn \& Hagedoorn, 1997).

In this project, we hope to learn something about the ways in which members of the First2 Network are connected and share information. To achieve this, we ask that you, an actor in this system, identify up to ten (10) other members of the Network (associates) with whom you communicate on issues that arise in the course of your work in the Network. Please note that you are not required to populate all ten fields, but we ask that you please identify at least one (1) associate.

Further, we ask that you qualitatively assign a code to each associate whose name you provide. These codes describe your level of engagement with each associate, ranging from a code of 1 for less strong relationships to 5 for strong collaborative ties.

This system of ranking from 1-5 follows from research conducted by Hogue in 1993 and Borden \& Perkins in 1998 and 1999. The levels of collaboration can be summarized as follows: 1. Networking

- Aware of Organization, Little Communication, Loosely Defined Roles, Independent Decision Making

2. Cooperation

- Share information, Formal Communication, Somewhat Defined Roles, Independent Decision Making

3. Coordination

- Share Information Frequently, Defined Roles, Some Shared Decision Making

4. Coalition

- Frequent Communication, Shared Resources, Shared Decision Making

5. Collaboration

- Frequent Communication, Shared Resources, and Mutual Trust. Coordination on Most or All Decision Making

Full descriptive characterizations of each category can be found in their publications, or at this website:

http://www.psnpaloalto.com/wp/wp-content/uploads/2011/04/PSN_Levels-of-CollaborationScale_survey.pdf

Additional instructions:

In the "Prior Acquaintance" column, please indicate with a checkmark those associates with whom you were in contact prior to your affiliation with the First2 Network.

In the "Key Contact" column, please indicate at most one (1) associate whom you consider to be of exceptional importance - in terms of resources, information, or guidance provided - to your role in the First2 Network.

\begin{tabular}{|c|c|c|c|}
\hline & \multicolumn{2}{|c|}{ Name } & \\
\hline & First Name & Last Name & \\
\hline Associate \#1 & & & \\
\hline Associate \#2 & & & \\
\hline Associate \#3 & & & \\
\hline Associate \#4 & & & \\
\hline Associate \#5 & & & \\
\hline Associate \#6 & & & 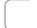 \\
\hline Associate \#7 & & & \\
\hline Associate \#8 & & & \\
\hline Associate \#9 & & & \\
\hline Associate \#10 & & & \\
\hline
\end{tabular}




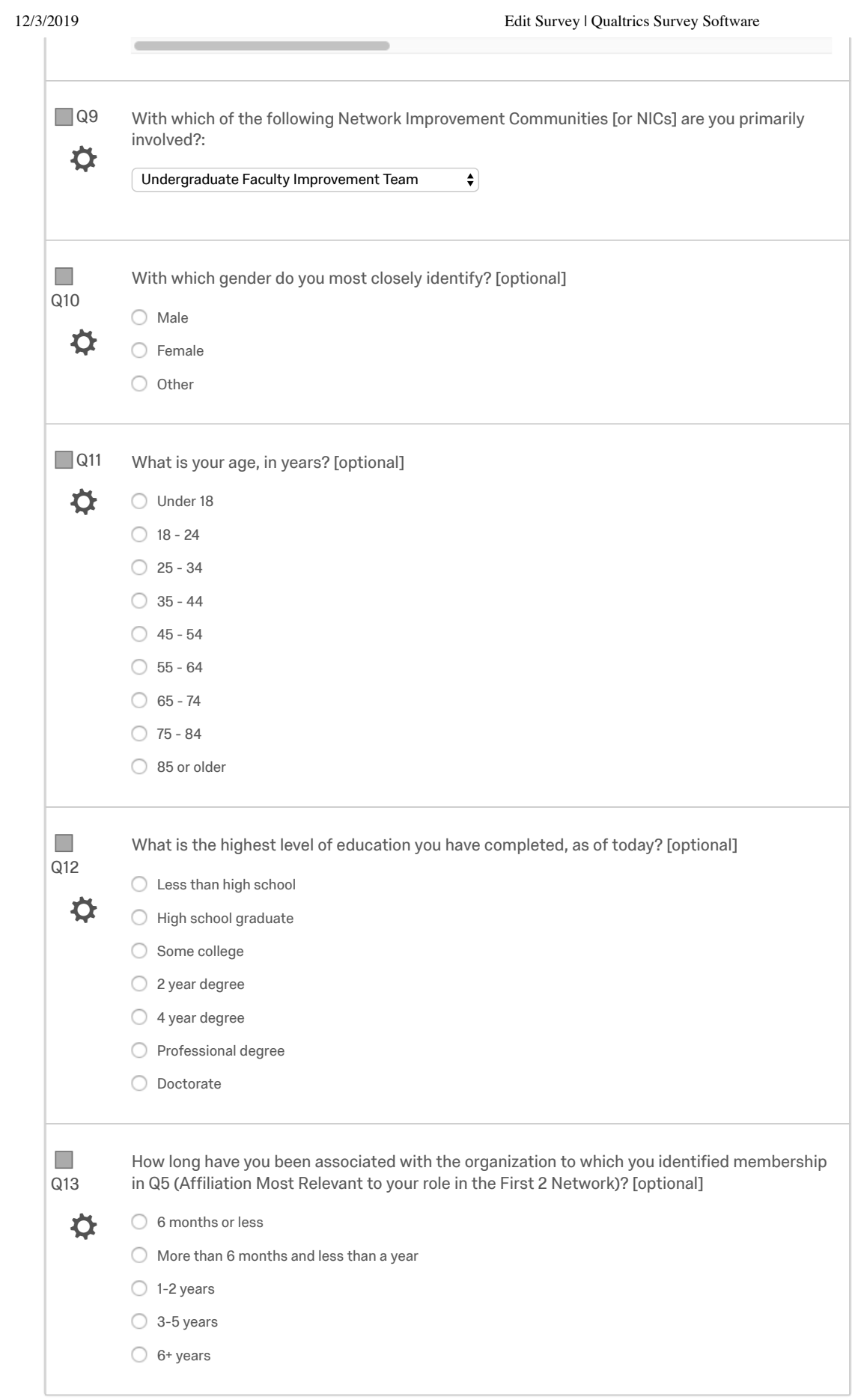

Add Block

\begin{tabular}{l|ll}
4 & End of Survey & Survey Termination Options...
\end{tabular}

https://wvu.ca1 .qualtrics.com/Q/EditSection/Blocks?SurveyID=SV_7WzJLoumXpbiMWV 


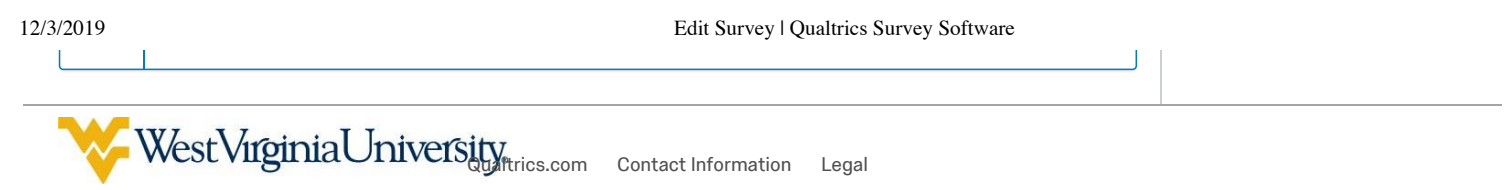

